KESANS : International Journal Of Health and Science

e-ISSN : 2808-7178, p-ISSN : 2808-7380

Web : http://kesans.rifainstitute.com/index.php/kesans/index

\title{
Understanding The Diagnosis and Treatment of Conjunctival Caviler Hemangioma
}

\author{
Baiq Ananda Audia Arsiazi \\ Faculty of Medicine, University of Mataram, Indonesia \\ audia18arsiazi@gmail.com
}

\begin{abstract}
Article Information
Accepted: 02 December 2021

Submitted: 31 December 2021

Online Publish: 20

January 2022
\end{abstract}

\begin{abstract}
Conjunctival hemangiomas are rare tumors that present at birth with reddish lesions that can grow in size over the next few months and spontaneously involute by 4-5 years of age. Symptoms of this tumor can be asymptomatic or can also cause visual disturbances if it has grown to be large. Capillary hemangiomas are more common in women and in premature or low birth weight infants. Both are associated with cardiorespiratory and hematological disorders. This tumor rarely attacks the age of over 60 years, but is more common in newborns. The diagnosis of hemangioma in addition to clinical symptoms, can also be established by supporting examinations. Treatment or management of these tumors depends on the presumptive diagnosis, size, growth, extent of the lesion, and the nature of the tumor.
\end{abstract}

Keywords : Conjunctival Capillary Hemangioma; Capillary Hemangioma of Conjunctiva; Capillary Hemangioma;
Baiq Ananda Audia Arsiazi /Understanding The Diagnosis and Treatment of Conjunctival Caviler Hemangioma/Vol. 1, No. 4, January 2022

https://doi.org/10.54543/kesans.v1i4.33 



\section{Introduction}

Orbital hemangioma is a congenital tumor that contains normal tissue in the form of blood vessels in the orbital area. In 1988, Mulliken divided hemangiomas into two types, namely the capillary type and the cavernous type. Then, in general, based on the location, the experts refined the classification of the hemangioma into three types, namely capillary hemangioma (superficial hemangioma), cavernous hemangioma (deep hemangioma), and mixed hemangioma (Herdiana, 2017). Capillary hemangiomas generally appear on the skin and rarely on the visceral organs. The shape varies from soft, bright red nodules to purple or plaque-shaped that has been visible since birth, or soon after birth, grows rapidly, and spontaneous involution occurs usually at the age of 5 years (Nafianti, 2016). Capillary hemangiomas often occur on the eyelids but rarely on the conjunctiva. Capillary hemangioma is a benign vascular tumor that results from the proliferation of endothelial cells. Its incidence is reported to be about $1 \%-2 \%$ of all benign conjunctival growths. It usually appears a few weeks after birth, grows over several months, then regresses spontaneously over years (S Padmanaban, P Sumathi and Dharmendra1, 2017). Conjunctival capillary hemangiomas with small lesions still require close monitoring and depend on the presumptive diagnosis, size, growth, lesion area, and the nature of the tumor (Nattis et al., 2017).

\section{Research Method}

The writing of this article uses various types of sources that come from scientific journals. Source searches were conducted on the online portals of journal publications such as the National Center for Biotechnology Information / NCBI (ncbi.nlm.nih.gov), Google Scholar (scholar.google.com), and Medscape. The keywords used are "Capillary Hemangioma" and "Conjunctiva Capillary Hemangioma".

\section{Discussion}

\section{Definition and Pathophysiology}

Conjunctival capillary hemangioma is a rapidly growing tumor originating from endothelial cells. However, most of the reported cases of conjunctival hemangioma are congenital, asymptomatic, and early growth at the age of 7 years (Nattis et al., 2017).

Capillary hemangioma is a benign vascular eyelid tumor that commonly occurs in children. About $1 \%-4 \%$ of all births, but is more common in premature babies. Appears several weeks after birth, usually skin, subcutaneous, or orbital lesions. In a series of 1463 cases of conjunctival tumors encountered, only 10 were found in capillary hemangiomas (S Padmanaban, P Sumathi and Dharmendra1, 2017).

Until now, the pathogenesis of hemangiomas is still unknown. Although growth factors, hormones, and mechanical influences are thought to be the cause of abnormal proliferation of hemangioma tissue, the main cause of defects in hemangiogenesis is still unclear. And it has not been proven to date about the influence of genetics. Skin vascularization begins to form on the 35th day of gestation, which continues for several months after birth. Maturation of the vascular system occurs in the 4th month after birth. 
Angiogenic factors may have an important role in the proliferative and involution phase of hemangiomas. The rapid endothelial growth in hemangiomas is similar to the capillary proliferation in tumors. Endothelial proliferation is affected by angiogenic agents. Angiogenic action works in two ways, namely, directly influencing vascular endothelial mitosis and indirectly affecting macrophages, mast cells, and helper $\mathrm{T}$ cells.

Heparin released by macrophages stimulates endothelial cell migration and capillary growth. In addition, heparin itself acts as an angiogenic agent. This effect of angiogenesis is inhibited by the presence of protamine, cartilage, and some corticosteroids. The concept of corticosteroid inhibition is applied to the treatment of several types of hemangioma in the involution phase.

Angioplasty, one of the internal fragments of plasminogen, is a potent and specific inhibitor for endothelial proliferation. Macrophages produce stimulators or inhibitors of angiogenesis. In the proliferative phase, the hemangioma tissue is infiltrated by macrophages and mast cells, while in the involution phase there is an infiltration of monocytes.

It is estimated that macrophage infiltration is influenced by Monocyte chemoattractant protein-1 (MCP-1), a glycoprotein that acts as a chemotaxis mediator. This substance is produced by vascular smooth muscle cells in the proliferative phase but is not produced by hemangiomas in the involution phase or vascular malformations. The presence of MCP-1 can be down-regulated by dexamethasone and interferon-alpha. Interferon-alpha has been shown to inhibit endothelial migration induced by chemotactic stimuli. This provides an additional effect of interferon-alpha in reducing the number and activity of macrophages. The above evidence explains the effect of dexamethasone and interferon-alpha on hemangiomas in the proliferative phase (Chang and Estes, 2014).

\section{Clinical Manifestations and Investigations}

Symptoms and signs are usually mostly asymptomatic or cause mild eye irritation to visual disturbances, there are small lumps, and spontaneous bleeding or bloody tears occur. In addition, it is followed by a lump in the blood vessels that is benign, slowly progressive, bright red in color with sometimes unclear edges, a reddish mass, stemmed, smooth, moving with the surface showing many blood vessels. Usually round, nodular, polypoid, or lobed, can grow and reach very large masses. It can be found as an isolated lesion or associated with other ocular capillary hemangiomas or systemic diseases such as the syndrome Sturge-Weber (Chauhan et al., 2017).

The diagnosis of hemangioma in addition to clinical symptoms can also be established by supporting examinations. Investigations are indicated when the clinical diagnosis is in doubt, prevents complications, or when surgery is imminent. Ultrasonography is useful in differentiating hemangiomas from deep dermal or subcutaneous structures, such as cysts or lymph nodes. Ultrasound in general has limitations for evaluating the size and spread of hemangiomas. It is also said that Doppler ultrasound $(2 \mathrm{kHz}$ ) can be used for high vessel density (more than 5 vessels/m2) and arterial crest changes. Examination using this tool is a sensitive and specific examination 
to identify an infantile hemangioma and distinguish it from other soft tissue masses. MRI is the imaging modality of choice because it is able to determine the location and spread of both cutaneous and extracutaneous hemangiomas. MRI can also help differentiate proliferating hemangiomas from other high-flow vascular lesions (eg arteriovenous malformations). Hemangiomas in the involution phase give the appearance of low-flow vascular lesions (eg venous malformations). In centers that do not have MRI facilities, CT scans can be used, although this method is not able to describe characteristics of blood flow. The use of contrast can help differentiate hemangiomas from other malignancies or masses that resemble hemangiomas.

Plain radiographs, such as X-rays, can still be used to see if hemangiomas are interfering with the airway. A biopsy is required when there is doubt about the diagnosis or to exclude caposiform hemangioen do the lioma or malignancy. The immunohistochemical examination can help establish the diagnosis. Complications that can occur in the biopsy are bleeding (Nafianti, 2010).

\section{Management}

Based on the above description, the management of conjunctival hemangioma depends on the presentation, growth, and extent of the tumor. Most hemangiomas that have not experienced complications received conservative therapy, either capillary, cavernous, or mixed hemangiomas. This is because most of these lesions will undergo spontaneous involution. Many cases of hemangioma who received conservative therapy had better results than surgical therapy both functionally and cosmetically. The mechanism of propranolol therapy in capillary hemangiomas is thought to be in three ways, namely a vasoconstrictive effect, decreasing the expression of genes vascular endothelial growth factor (VEGF), and basic fibroblast growth factor (bFGF), and inducing apoptosis in capillary endothelial cells. At what phase propranolol is effective given, is still being debated. The dose used is $2 \mathrm{mg} / \mathrm{kg} \mathrm{BW} /$ day. Shah (2014) in his research started a propranolol dose of $0.33 \mathrm{mg} / \mathrm{kg}$ BW given 8 hours a day for 1 week and increased to $0.67 \mathrm{mg} / \mathrm{kg} \mathrm{BW}$ to the recommended dose of $2 \mathrm{mg} / \mathrm{kg} / \mathrm{BW}$. The duration of propranolol treatment is recommended for a minimum of 6 months and is recommended to be effective for 12 months (Herdiana, 2017). In addition to the medical treatment described above, there is also surgical excision. Indications for surgical excision are hemangiomas that grow progressively, hemangiomas that experience repeated infection, hemangiomas whose surface resonates, so that they are feared to be accompanied by malignancy, cosmetically disturbing, hemangiomas that fail medical treatment, and stemmed hemangiomas (Das, Raj and Bajaj, 2020).

\section{Conclusion}


Conjunctival capillary hemangioma is a rapidly growing tumor originating from endothelial cells. Capillary hemangioma is a benign vascular eyelid tumor that commonly occurs in children. Until now, the pathogenesis of hemangiomas is still unknown. However, angiogenic factors may have an important role in the proliferative and involution phase of hemangiomas. Management of conjunctival hemangiomas depends on the presentation, growth and extent of the tumor. 
Chang, T. C. and Estes, R. (2014) 'Beta blocker treatment of infantile conjunctival hemangiomas - Observations from 2 cases’, Journal of AAPOS, 18(1), pp. 80-82. doi: 10.1016/j.jaapos.2013.09.013.

Chauhan, A. et al. (2017) 'Hemangioma Kapiler Konjungtiva: Pertumbuhan Permukaan Mata Langka', (April).

Das, D., Raj, S. J. S. and Bajaj, M. S. (2020) 'Acquired sessile conjunctival capillary haemangioma in an adult managed with topical timolol', BMJ Case Reports, 13(8). doi: 10.1136/bcr-2020-237547.

Herdiana (2017) Onkologi Mata, Journal of Chemical Information and Modeling.

Nafianti, S. (2016) 'Hemangioma pada Anak', Sari Pediatri, 12(3), p. 204. doi: 10.14238/sp12.3.2010.204-10.

Nattis, A. et al. (2017) 'Conjunctival Capillary Hemangioma', Cureus, 9(11), pp. 29-32. doi: $10.7759 /$ cureus.1892.

S Padmanaban, P Sumathi, P. K. and Dharmendra1, R. P. (2017) 'congenital capillary hemangioma arising from palpebral conjunctiva of a neonate', $B M C$ Ophthalmology, 17(1), p. 1. doi: 10.4103/ijo.IJO.

\section{Copyright holder:}

Baiq Ananda Audia Arsiazi (2022)

First publication right:

KESANS: International Journal Health and Science 